\title{
On the Statistical Characterization of the Discrete Fourier Transform of Noisy Space Vectors
}

\author{
Diego Bellan \\ Department of Electronics, Information and Bioengineering \\ Politecnico di Milano \\ Milan, Italy \\ diego.bellan@polimi.it
}

\begin{abstract}
This paper investigates the statistical effects of additive noise on the magnitude of the space-vector spectral lines estimated through a discrete Fourier transform (DFT). In fact, the space vector is a well-known and effective tool for monitoring power quality issues in modern power systems. In many practical cases waveform distortion requires a DFT in order to extract the fundamental component and the harmonic/interharmonic content. In particular, the space vector shape (on the complex plane) of the fundamental component provides information such as voltage dips, whereas space-vector spectral lines provide information related to waveform distortion. Additive noise mainly impacts on low-magnitude spectral lines which require a statistical characterization. Conventional results available in the literature cannot be used in a straightforward way because a space vector is a complex-valued function, therefore special care is needed for proper interpretation and use of its properties in the frequency domain. In the paper, the probability density function, the mean value and the variance of the magnitude of space-vector DFT spectral lines are derived in closed form. Analytical results are validated by means of numerical simulations.
\end{abstract}

Keywords-power quality, distorted power systems, additive noise, discrete Fourier transform, Clarke transformation, space vectors

\section{INTRODUCTION}

Three-phase electrical power systems nowadays are characterized by a widespread use of nonlinear and time-varying components [1]-[4]. As a consequence, power quality issues are gaining more and more importance for a proper system characterization. In recent years it was shown that the space vector tool [5] can be effectively exploited to detect and characterize some specific power quality issues (e.g., voltage dips [6]). To this aim, the parameters of the space vector shape on the complex plane (i.e., the axes and the inclination angle of the elliptical space-vector trajectory) require a careful evaluation. However, in many practical cases the waveform distortion and the additive noise prevent the possibility of such accurate evaluations. Much more accurate evaluations can be obtained by transforming the space vector into the frequency domain through the discrete Fourier transform (DFT) [6]-[8]. In fact, the use of DFT provides two main advantages. First, the fundamental component of the space vector can be accurately estimated. This leads to accurate estimation of the parameters of the space vector shape on the complex plane, and the corresponding detection and classification of voltage dips. Second, the spectral content of the space vector can be estimated by the DFT spectral lines. Thus, the effects of waveform distortion can be accurately measured. Measurements are always affected by additive noise. However, additive noise has larger impact on spectral lines with lower magnitude. Therefore, it is expected a negligible impact on the space-vector fundamental component, and larger impact on space-vector harmonics/interharmonics. Noise propagation through DFT is a well-known subject in the technical literature (e.g., [9]-[11]). However, since a space vector is a complex-valued function, special care must be used in the interpretation of spectrum components and the application of DFT. To the Author's knowledge, in the existing literature there is a lack of analysis regarding such points. In Section II the basic properties of spacevector Fourier series expansion and DFT are recalled. In Section III the statistical effects of additive input noise on the DFT estimation of low-amplitude space-vector components (e.g., corresponding to higher harmonic order) are presented. In particular, the probability density function, the mean value, and the variance of spectral lines magnitude are derived in closed form. Numerical validation is presented in Section IV where a specific example of a distorted and noisy space vector is simulated. Concluding remarks are drawn in Section V.

\section{FourIER SERIES AND DFT OF SPACE VECTORS}

Space vector is a powerful mathematical tool for the analysis of three-phase circuits under arbitrary time-domain conditions. Existing literature has already addressed transient and sinusoidal steady-state analyses in terms of space vectors. Much less attention, however, was devoted to the analysis under distorted conditions. In this Section it will be shown that the Fourier series expansion holds for space vectors, and its relationship with the symmetrical components transformation (SCT) will be put into evidence. Moreover, the use of the DFT will be shown in order to estimate the Fourier components of a space vector.

Let us consider three time-domain periodic variables $x_{a}(t), x_{b}(t), x_{c}(t)$ with common fundamental period $T$. We assume that each variable can be expanded in a Fourier series. Thus, by adopting the complex form, we can write:

$$
x_{u}(t)=\sum_{k=-\infty}^{+\infty} X_{u, k} \exp \left(j k \omega_{0} t\right), \quad u=a, b, c
$$

where $\omega_{0}=2 \pi / T$, and the complex Fourier coefficients are given by:

$$
X_{u . k}=\frac{1}{T} \int_{0}^{T} x_{u}(t) \exp \left(-j k \omega_{0} t\right) d t
$$


In order to introduce the space vector $\bar{x}(t)$ related to the phase variables $x_{a}(t), x_{b}(t), x_{c}(t)$, the Clarke transformation must be applied first [5]-[8], [12]-13]:

$$
\left[\begin{array}{l}
x_{\alpha} \\
x_{\beta} \\
x_{0}
\end{array}\right]=\sqrt{\frac{2}{3}}\left[\begin{array}{ccc}
1 & -\frac{1}{2} & -\frac{1}{2} \\
0 & \frac{\sqrt{3}}{2} & -\frac{\sqrt{3}}{2} \\
\frac{1}{\sqrt{2}} & \frac{1}{\sqrt{2}} & \frac{1}{\sqrt{2}}
\end{array}\right] \cdot\left[\begin{array}{l}
x_{a} \\
x_{b} \\
x_{c}
\end{array}\right]=\mathbf{T}_{\mathbf{0}} \mathbf{x}
$$

where $\mathbf{T}_{\mathbf{0}}$ is an orthogonal matrix. The space vector is defined as

$\bar{x}(t)=x_{\alpha}(t)+j x_{\beta}(t)=\sqrt{\frac{2}{3}}\left(x_{a}(t)+\alpha x_{b}(t)+\alpha^{2} x_{c}(t)\right)$

where $\alpha=\exp (j 2 \pi / 3)$.

Notice that since $x_{a}(t), x_{b}(t), x_{c}(t)$ are periodic functions, from (3) we obtain that also $x_{\alpha}(t)$ and $x_{\beta}(t)$ are periodic functions whose Fourier series expansion can be written:

$$
x_{v}(t)=\sum_{k=-\infty}^{+\infty} X_{v, k} \exp \left(j k \omega_{0} t\right), \quad v=\alpha, \beta
$$

where the Fourier coefficients are evaluated through the expression corresponding to (2).

By taking into account (4), it follows that also the space vector can be expanded in Fourier series:

$$
\bar{x}(t)=\sum_{k=-\infty}^{+\infty} X_{k} \exp \left(j k \omega_{0} t\right)
$$

where

$$
\begin{gathered}
X_{k}=\frac{1}{T} \int_{0}^{T} \bar{x}(t) \exp \left(-j k \omega_{0} t\right) d t= \\
=X_{\alpha, k}+j X_{\beta, k}
\end{gathered}
$$

The result in (7) allows to highlight the main property of the space-vector Fourier series, i.e., $X_{-k} \neq X_{k}^{*}$. In fact, since the space vector is a complex-valued function, unlike the realvalued functions in (1) and (5), the Fourier coefficients for negative frequencies are different from the complex conjugate of the Fourier coefficients for positive frequencies [12]. The main consequence is that when the DFT algorithm is applied to evaluate the frequency content of a space vector, the two-sided spectrum must be considered (i.e., positive and negative frequencies) instead of the one-sided spectrum as for real-valued waveforms [7]-[11]:

$$
X_{k}=\frac{1}{N_{S} N P S G} \sum_{n=0}^{N_{S}-1} \bar{x}\left(n T_{S}\right) w\left(n T_{s}\right) \exp \left(-j \frac{2 \pi k n}{N_{S}}\right)
$$

where NPSG is the Normalized Peak Signal Gain related to the window function $w(t)$ selected to reduce the frequency-domain spectral leakage phenomenon. For example, NPSG $=1$ for the rectangular window, and NPSG $=0.50$ for the widely used Hann window. Moreover, in (8) $N_{s}$ is the number of samples and $T_{s}$ is the sampling period.

It is interesting to establish the relationship between the space-vector Fourier coefficients and the positive/negative components resulting from the SCT [8], [12], [14]-[15]. To this aim, since the SCT refers to phasor quantities, we consider a specific frequency component $\omega=k \omega_{0}$. According to the SCT, the phasors (rms) of the phase variables can be expressed as functions of the positive, negative, and zero-sequence components as [4]:

$$
\left[\begin{array}{l}
X_{a} \\
X_{b} \\
X_{c}
\end{array}\right]=\frac{1}{\sqrt{3}}\left[\begin{array}{ccc}
1 & 1 & 1 \\
\alpha^{2} & \alpha & 1 \\
\alpha & \alpha^{2} & 1
\end{array}\right]\left[\begin{array}{l}
X_{p} \\
X_{n} \\
X_{0}
\end{array}\right]
$$

The time-domain waveforms can be expressed in terms of the related phasors as:

$$
\left[\begin{array}{l}
x_{a}(t) \\
x_{b}(t) \\
x_{c}(t)
\end{array}\right]=\frac{1}{\sqrt{2}}\left(\left[\begin{array}{l}
X_{a} \\
X_{b} \\
X_{c}
\end{array}\right] \exp (j \omega t)+\left[\begin{array}{l}
X_{a}^{*} \\
X_{b}^{*} \\
X_{c}^{*}
\end{array}\right] \exp (-j \omega t)\right)
$$

By substituting (9)-(10) into (4) we obtain:

$$
\bar{x}(t)=X_{p} \exp (j \omega t)+X_{n}^{*} \exp (-j \omega t)
$$

The simple result in (11) is of paramount importance in the interpretation of the general expression (6). In fact, from (11) we have that a specific frequency component $\omega=k \omega_{0}$ results in a space vector with two components: a positive frequency component $k \omega_{0}$ with complex coefficient given by the positive sequence phasor $X_{p}$, and a negative frequency component $-k \omega_{0}$ with complex coefficient given by the complex conjugate of the negative sequence phasor $X_{n}$. Thus, in the general Fourier series expansion (6), the Fourier coefficients $X_{k}$ and $X_{-k}$ are equal to the positive and complex-conjugate negative sequence phasors at $\omega=k \omega_{0}$.

\section{DFT OF NoIsy SPACE Vectors}

When the DFT (8) is applied to a noisy space vector (4) resulting from additive zero-mean white noise with standard deviation (STD) $\sigma_{n}$ in the phase variables $x_{a}(t), x_{b}(t), x_{c}(t)$, each estimated Fourier coefficient $X_{k}$ should be treated as a random variable (RV). In [9]-[11], [16]-[18] it was shown that the real and imaginary parts of $X_{k}$ can be approximated by unbiased and uncorrelated Gaussian RVs with equal variances:

$$
\sigma^{2}=E N B W \frac{\sigma_{n}^{2}}{N_{S}}
$$

where ENBW is the equivalent noise bandwidth of the selected window function (e.g., ENBW=1 for rectangular window, $\mathrm{ENBW}=1.50$ for the Hann window). It follows that the magnitude $\left|X_{k}\right|$ of the $k$-th frequency component of the space vector (i.e., the magnitude of the positive sequence phasor for positive $k$ and the magnitude of the negative sequence phasor for negative $k$ ) is a RV with Rice distribution [19]-[20]. The probability density function (PDF) is given by:

$$
f(y)=\frac{y}{\sigma^{2}} \exp \left(-\frac{y^{2}+A^{2}}{2 \sigma^{2}}\right) I_{0}\left(\frac{y A}{\sigma^{2}}\right)
$$

where $y$ denotes the $\mathrm{RV}\left|X_{k}\right|, A$ denotes the noise-free value of $\left|X_{k}\right|$, and $I_{0}$ is the modified Bessel function of the first kind with order zero. The mean value and the variance of $\left|X_{k}\right|$ are given by $[8]$ :

$$
\mu_{X}=\sigma \sqrt{\frac{\pi}{2}} L_{1 / 2}\left(-\frac{A^{2}}{2 \sigma^{2}}\right)
$$




$$
\sigma_{X}^{2}=2 \sigma^{2}+A^{2}-\frac{\pi \sigma^{2}}{2} L_{1 / 2}^{2}\left(-\frac{A^{2}}{2 \sigma^{2}}\right)
$$

where $L$ is the Laguerre polynomial.

\section{NUMERICAL VALIDATION}

Let us consider a three-phase system under distorted conditions, and three phase variables $x_{a}(t), x_{b}(t), x_{c}(t)$ (i.e., phase voltages or currents) whose space vector $\bar{x}(t)$, according to (6), is characterized by the amplitude spectrum shown in Fig. 1 (i.e., the set of the magnitudes of the Fourier coefficients $X_{k}$ as function of the harmonic index $k$ ). As mentioned above, the amplitude spectrum is not symmetrical with respect to the frequency index. In fact, positive index values correspond to positive sequence components, whereas negative index values correspond to negative sequence components. Notice that it was assumed harmonic components different from zero only for $k=$ $+1,-2,+4,-5,+7,-8, \ldots$ (i.e., the so-called characteristic harmonics). Indeed, it can be shown that this is a consequence of the common case where the waveforms of the three phase variables are overlapping and displaced each other by one third of the period $T$ [12].

In Fig. 1 the fundamental component at $k=+1$ was assumed with magnitude 100 , whereas the other components have decreasing amplitude with the harmonic index (notice the logarithmic scale for the magnitude). When additive noise is considered, it is expected that its impact is larger for harmonic components with smaller amplitude. For this reason, the harmonic component with unit magnitude corresponding to $k=$ -8 was considered to investigate the effects of additive noise. Fig. 2 shows the behavior of the PDF of the RV $\left|X_{-8}\right|$ obtained by considering additive zero-mean Gaussian noise with $\sigma_{n}=1$ which was added to each of the three phase variables in the time domain. The analytical result (13) (solid lines) is compared with numerical results obtained through repeated-run simulations (dashed lines). The effect of a different time window is also shown in the figure, i.e., using a window with ENBW larger than one (the Hann window in this case, blue curves) results in a PDF with larger spread with respect to the reference case ENBW=1 (i.e., the rectangular window, black curves).

Fig. 3 shows the behavior of the mean value of the RV $\left|X_{-8}\right|$ as a function of the STD $\sigma_{n}$ of the additive input noise. The analytical result given in (14) (solid lines) is compared with numerical results obtained by repeated-run simulations (dashed lines). As it was expected, increasing additive noise level results in increasing bias of $\left|X_{-8}\right|$. According to (12), selection of a time window with larger ENBW and/or using a smaller number of samples $N_{S}$ results in larger value of $\sigma$ leading to larger bias.

Finally, similarly to Fig. 3, Fig. 4 shows the STD of the RV $\left|X_{-8}\right|$ as a function of the STD $\sigma_{n}$ of the additive input noise. The analytical result given in (15) (solid lines) is compared with numerical results obtained by repeated-run simulations (dashed lines). As it was expected, increasing additive noise level results in increasing STD of $\left|X_{-8}\right|$. Also in this case, according (12), selection of a time window with larger ENBW and/or using a smaller number of samples $N_{S}$ results in larger values of the output STD.

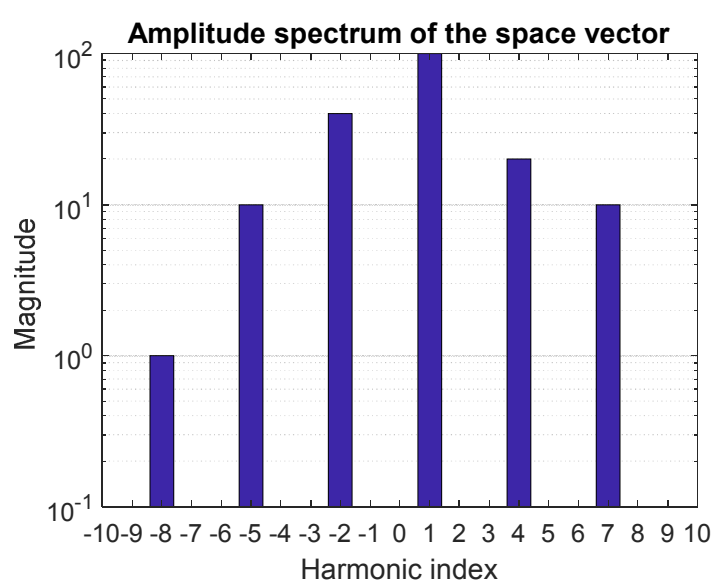

Figure 1. Amplitude spectrum of the example space vector defined in the text.

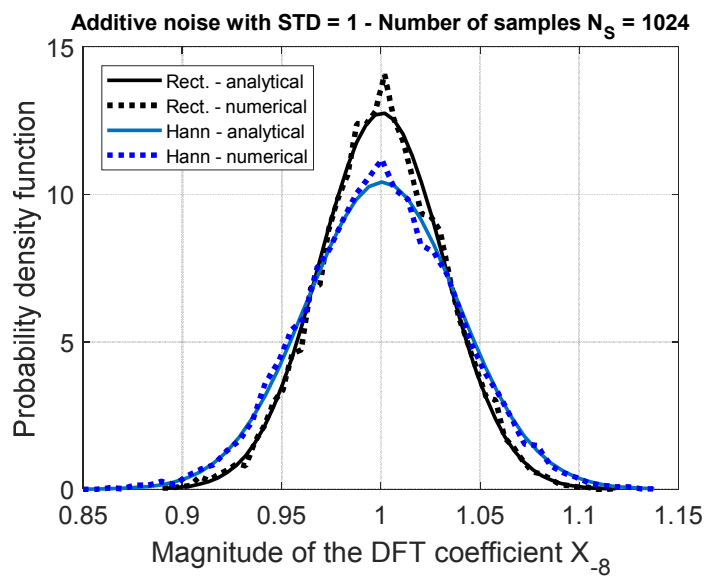

Figure 2. PDF of the RV $\left|X_{-8}\right|$ when additive input noise with unit STD is considered. Rectangular and Hann windows are compared. Solid lines refer to the analytical result (13), whereas dashed lines refer to repeated-run simulations.

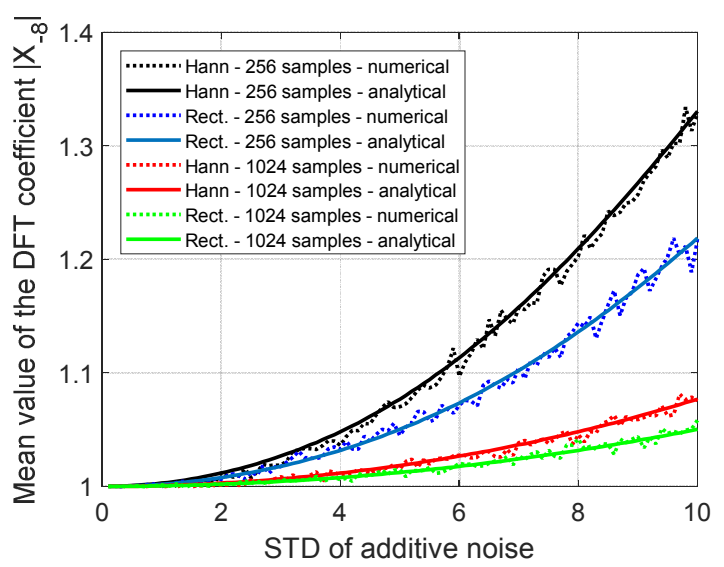

Figure 3. Behavior of the mean value of the $\operatorname{RV}\left|X_{-8}\right|$ as a function of the STD $\sigma_{n}$ of the additive input noise. Two different windows (rectangular and Hann) and two number of samples (256 and 1024) were considered for comparison. 


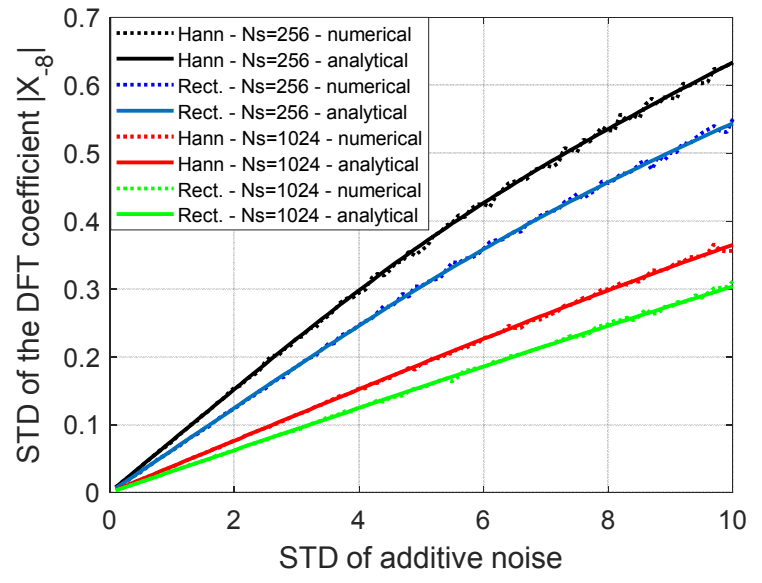

Figure 4. Behavior of the standard deviation of the $\mathrm{RV}\left|X_{-8}\right|$ as a function of the standard deviation $\sigma_{n}$ of the additive input noise. Two different windows (rectangular and Hann) and two number of samples (256 and 1024) were considered for comparison.

\section{CONCLUSION}

The space vector tool has proved to be very effective in the analysis of distorted and noisy three-phase systems. Indeed, it allows the analysis under general working conditions. Under distorted conditions the relationship between each space-vector harmonic component and the related SCT components was shown. Digital techniques for spectrum evaluation, such as the DFT, can be readily used for distorted and noisy space vectors. It was shown, however, that space vectors require evaluation of two-side spectra instead of one sided. Analytical expressions for the mean value and standard deviation of measured DFT components were obtained under noisy conditions. This point is crucial in the estimate of low-amplitude harmonic components. Moreover, the statistical effects of the choice of different time windows and number of samples have been clearly put into evidence.

\section{REFERENCES}

[1] M. H. J. Bollen, Understanding Power Quality Problems, New York: IEEE Press, 2000.

[2] M. Olofsson and U. Grape, "Voltage quality in the context of EMC," in Proc. International Symposium on Electromagnetic Compatibility, 2009, p. 241.

[3] D. Bellan, G, Spadacini, E. Fedeli, and S. A. Pignari, "Space-frequency analysis and experimental measurement of magnetic field emissions radiated by high-speed railway systems," IEEE Trans. Electromagn. Compat., vol. 55, no. 6, pp. 1031-1042, 2013.

[4] D. Bellan, S. A. Pignari, G. Superti-Furga, "Consistent circuit technique for zero-sequence currents evaluation in interconnected single/three- phase power networks," Journal of Electrical Systems, vol. 12, no. 2, pp. 230-238, 2016.

[5] J. Aller, A. Bueno, and T. Paga, "Power system analysis using space vector transformation," IEEE Transactions on Power Systems, vol. 17, no. 4, pp. 957-965, November 2002.

[6] V. Ignatova, P. Granjon, and S. Bacha, "Space vector method for voltage dips and swells analysis," IEEE Trans. on Power Delivery, vol. 24, no. 4, pp. 2054 - 2061, 2009.

[7] F. A. S. Neves, H. E. P. de Souza, F. Bradaschia, M. C. Cavalcanti, M. Rizo, F. J. Rodriguez, "A space-vector discrete Fourier transform for unbalanced and distorted three-phase signals," IEEE Trans. on Industrial Electronics, vol. 57, no. 8, pp. 2858-2867, Aug. 2010.

[8] D. Bellan, "On the statistics of noisy space vector in power quality analysis," International Journal of Engineering and Technology, vol. 8, no. 5, pp. 2177-2183, 2016.

[9] O. M. Solomon, "The use of DFT windows in signal-to-noise ratio and harmonic distortion computations," IEEE Trans. Instrum. Meas., vol. 43, pp. 194-199, April 1994.

[10] D. Bellan, A. Gaggelli, and S. A. Pignari, "Noise effects in time-domain systems involving three-axial field probes for the measurement of nonstationary radiated emissions," IEEE Trans. on Electromagn. Compat., vol. 51, no. 2, pp. 192-203, 2009.

[11] D. Bellan and S. A. Pignari, "Statistical properties of real-time amplitude estimate of harmonics affected by frequency instability," Journal of Electrical Engineering (JEEEC), vol. 67, no. 4, pp. 292-298, 2016.

[12] G. Superti-Furga, S. Barcellona, E. Tironi, "Space-vector approach in three-phase unbalance and distortion analysis," in Proc. of $17^{\text {th }}$ International Conference on Harmonics and Quality of Power (ICHQP), 16-19 Oct. 2016, pp. 721-726.

[13] M. Owen, "Transient analysis using component transforms," in Proc. of 2011 IEEE Power Engineering and Automation Conference, 2011, pp. 49.

[14] G. C. Paap, "Symmetrical components in the time domain and their application to power network calculations," IEEE Trans. on Power Systems, vol. 15, no. 2, pp. 522-528, May 2000.

[15] G. Chicco, P. Postolache, and C. Toader, Analysis of three-phase systems with neutral under distorted and unbalanced conditions in the symmetrical component-based framework, IEEE Trans. on Power Delivery, vol. 22, no. 1, pp. 674-683, 2007.

[16] D. Bellan, "Statistical properties of voltage unbalance factor in threephase power systems," International Journal of Applied Engineering Research, vol. 11, no. 15, pp. 8475-8479, Aug. 2016.

[17] D. Bellan, "Statistical characterization of harmonic emissions in power supply systems," International Review of Electrical Engineering, vol. 9, no. 4, pp. 803-810, 2014.

[18] D. Bellan, "Characteristic Function of Fundamental and Harmonic Active Power in Digital Measurements Under Nonsinusoidal Conditions," International Review of Electrical Engineering, vol. 10, no. 4, pp. 520$527,2015$.

[19] M. K. Simon, Probability Distributions Involving Gaussian Random Variables, Springer, 2002.

[20] D. Bellan and S. A. Pignari, "Statistical superposition of crosstalk effects in cable bundles," China Communications, vol. 10, no. 11, pp. 119-128, 2013 